\title{
Discovery of novel community-relevant small proteins in a simplified human intestinal microbiome
}

Hannes Petruschke

Christian Schori

Sebastian Canzler

Sarah Riesbeck

Anja Poehlein

Rolf Daniel

Daniel Frei

Tina Segessemann

Johannes Zimmerman

Georgios Marinos

Christoph Kaleta

Nico Jehmlich

Christian H. Ahrens

Martin von Bergen

\section{Video Byte}

Keywords: Small proteins (sProteins), SIHUMIx, human gut microbiome, proteogenomics, iPtgxDB, metatranscriptomics, metaproteomics, metabolic modeling, Microbiome

Posted Date: March 11th, 2021

DOl: https://doi.org/10.21203/rs.3.rs-318776/v1

License: (9) This work is licensed under a Creative Commons Attribution 4.0 International License. Read Full License 


\section{Abstract}

The human intestinal microbiota plays a central role in regulating metabolism and immunity. Disturbances in homeostasis can lead to dysbiosis, which is associated with various diseases. A recent study aimed to discover novel small proteins under 100 amino acids (sProteins) contributing to the composition of the human intestinal microbiota. Using a proteogenomics approach, researchers identified novel sProteins in the gut microbiota, focusing on species belonging to the simplified human intestinal microbiota (SIHUMIx), a lower-complexity model system for the intestinal microbiome. This approach identified 31 novel sProteins, with 30 of those supported by metatranscriptomics and 25 validated by synthetic peptides. Six of these novel sProteins were only identified in the SIHUMIX community, indicating a potentially important role in the organization of microbial communities. Two of the discovered sProteins have a potential antimicrobial function, and the gene for another is located in a region encoding several metabolic enzymes. This integrated experimental and bioinformatics-based workflow will aid in the discovery of novel sProteins, enabling new insights into the role of sProteins in bacterial community function. 